\title{
Mersalyl Acid
}

National Cancer Institute

\section{Source}

National Cancer Institute. Mersalyl Acid. NCI Thesaurus. Code C66098.

A mercurial diuretic. Mersalyl acid has been replaced by less toxic non-mercury containing diuretics. 